\title{
Introducing delay dynamics to Bertalanffy's spherical tumour growth model
}

\author{
J.A. Roberts ${ }^{\mathrm{a}, *}$, A. Al Themairi ${ }^{\mathrm{b}}$ \\ ${ }^{a}$ Department of Mathematics, University of Chester, Thornton Science Park, UK CH2 4NU \\ ${ }^{b}$ University of Princess Nourah bint Abdulrahman, Saudi Arabia
}

\begin{abstract}
We introduce delay dynamics to an ordinary differential equation model of tumour growth based upon von Bertalanffy's growth model, a model which has received little attention in comparison to other models, such as Gompterz, Greenspan and logistic models. Using existing, previously published data sets we show that our delay model can perform better than delay models based on a Gompertz, Greenspan or logistic formulation. We look for replication of the oscillatory behaviour in the data, as well as a low error value (via a Least-Squares approach) when comparing. We provide the necessary analysis to show that a unique, continuous, solution exists for our model equation and consider the qualitative behaviour of a solution near a point of equilibrium.
\end{abstract}

Keywords: delay differential equation, tumour growth

\section{Review of existing work}

In recent years there have been a number of articles focusing on models of tumour growth dynamics (for example, see texts by Gerlee [12], Bi and Ruan [4] as well as the text by Bodnar and Foryś [5], which we discuss later in more detail). One approach has been to consider a tumour as a population of cells and to use ordinary differential equations to model this population. Classical ordinary differential equation models include the Gompertz model

$$
V^{\prime}(t)=\alpha V(t)-\beta V(t) \ln V(t),
$$

the Logistic model

$$
V^{\prime}(t)=\alpha V(t)-\beta V^{2}(t),
$$

the Greenspan model

$$
V^{\prime}(t)=\alpha V(t)\left(1-\left(\frac{V(t)}{\beta}\right)^{\frac{2}{3}}\right),
$$

and the Bertalanffy model

$$
V^{\prime}(t)=\alpha V(t)^{\frac{2}{3}}-\beta V(t)
$$

\footnotetext{
* Corresponding author
} 
where $\alpha$ and $\beta$ are real, positive constants and $V(t)$ is the volume of the tumour at time $t$; the rate of change in volume $V^{\prime}(t)$ is defined to be the difference, at time $t$, between the rate of growth and the rate of degradation. These equations, and variations of them are discussed in detail within the paper by Marušić et al [14]. Exact solutions to these equations are easily obtained via standard techniques and all produce a sigmoidal-shaped solution curve.

Marušić et al [14] describes how these models can sometimes fail to be adequate matches for the data. Biological processes can include time delays within their functional responses. There are a number of texts available which discuss this in large detail. Examples include the books by Murray [15] (which introduces delay models in Chapter One) and Brauer \& Castillo-Chavez [9] (which introduces delay terms to population models in Chapter Three). For example, a delay-logistic model of the form

$$
V^{\prime}(t)=\alpha V(t)\left(1-\frac{V(t-\tau)}{\beta}\right),
$$

where $\alpha, \beta$ and $\tau$ are real, positive constants is considered.

The text by Smith [17] discusses techniques for establishing the existence of solutions to such models (such as the Method of Steps approach introduced originally by Bellman and Cooke [2]) and for investigating the qualitative behaviour of such solutions.

Bodnar and Foryś [5] provide a quick review of the previously cited ordinary differential equation models, with the exception of the Bertalanffy model (4) before introducing discrete, constant delays into terms within the models.

They first introduce a delay into the per capita growth rate by rewriting the models in the general form

$$
\frac{V^{\prime}(t)}{V(t)}=f(V(t))
$$

and then introducing a delay $\tau$ as follows:

$$
\frac{V^{\prime}(t)}{V(t)}=f(V(t-\tau))
$$

For each of these models, questions relating to the existence of continuous, positive solutions are answered and accompanied by a stability analysis.

A second set of delay equations are introduced in [5] by writing the differential equations models in the form

$$
V^{\prime}(t)=V(t) f(V(t))
$$

and introducing a discrete delay $\tau$ in the net proliferation rate as follows:

$$
V^{\prime}(t)=V(t-\tau) f(V(t-\tau)) .
$$

The authors' justification for such a formulation is based on the cell cycle; the paper by Byrne [10] discusses two ways in which the cell cycle can be modelled by delays in this net growth rate: (i) the time taken for cells to undergo mitosis, (ii) the time for changes in the proliferation rate to stimulate compensatory changes in apoptotic cell loss. The paper [5] introduces delays for the logistic, Gompertz and Greenspan models and includes derivations of results related to properties of solutions to the equations, such 
as existence and qualitative behaviour. Furthermore, for each model that the authors consider, they fit parameters to previously published data. The data, experimental data for Ehrlich Ascitis tumours in mice, is taken from the article by Schuster \& Schuster [16]. The purpose of this parameter fitting is (according to the authors) "to answer the question if, in the case with delay, the Gompertz model is the best one for describing early tumour growth".

The Bertalanffy model is not considered in the paper [5] and is discussed in comparatively far less literature than the logistic, Gompertz or Greenspan models. Gerlee's paper [12] provides an historical survey of ordinary differential equation approaches to modelling tumour growth and notes explicitly that the Bertalanffy model (4) has received considerably less attention than other models.

Ludwig von Bertalanffy first proposed this equation as a model of organism growth in the paper [3]. The model is constructed so that its parameters and form have a clear biological meaning. For a spherical collection of cells the growth rate is considered proportional to the surface area of the sphere (hence the $\alpha V^{\frac{2}{3}}(t)$ term) and the loss of mass due to cell death is proportional to the volume of the sphere (hence the $-\beta V(t)$ term). Gerlee [12] notes also that the Bertalanffy model can give a better fit than both the logistic and Gompertz models, citing a study by V.G. Vaidya \& F.J. Alexandro Jr [18] in which it is demonstrated that the Bertalanffy was a better fit than the logistic (2) or the Gompertz (1) models in 7 out of 10 cases studied, involving experimental mouse data.

Gerlee [12] presents a number of arguments to hypothesise why models such as the Gompertz model holds a dominating position within the ordinary differential equation approach to tumour modelling. He suggests the ease with which data can be fitted to the model as a practical reason and, as a theoretical reason, he suggests that the Gompertz model's ability to predict can be used as an argument to ignore the fact that the model's component parts may not be constructed from a biological viewpoint. Gerlee [12] concludes that the Bertalanffy model of tumour growth is the most suitable candidate to look at next if one wants to focus on a model that is both grounded in biological theory and can be fitted adequately to data via parameter estimation.

Thus, we are motivated to examine the Bertalanffy model and consider the hypothesis that a better fit to data may be obtained through the introduction of delay terms to the model. We will test this hypothesis by examining the following features in the model:

- do unique, continuous, positive solutions exist to the proposed model?

- is the model capable of producing solutions whose 'shape' replicates that seen in the data, when plotted? i.e. does the solution display oscillatory behaviour when such behaviour is shown to exist in the data?

- for the data sets published in [16], can parameter values be identified which produce error values (calculated via a Least Squares approach) that are smaller than or similar to those produced for the delay models examined in [5]? 


\section{Theoretical considerations}

We propose the following model as an extension of the Bertalanffy ordinary differential equation model:

$$
\begin{gathered}
V^{\prime}(t)=\alpha V^{\frac{2}{3}}\left(t-\tau_{1}\right)-\beta V\left(t-\tau_{2}\right), t \in[\tau, T), \\
V(t)=\varphi(t)>0, t \in[0, \tau],
\end{gathered}
$$

where $\alpha, \beta, T \in(0, \infty), \tau_{1}, \tau_{2} \in(0, T), \tau=\max \left\{\tau_{1}, \tau_{2}\right\}$ are fixed constants and $\varphi(t)$ is a real, positive, continuous function.

Our choice of two, possibly distinct delays is based on the following biological reasoning. $\tau_{1}$ represents the time taken for the cells to reach the dividing stage of their cycle. $\tau_{2}$ can be interpreted as the mean life expectancy of a single cell.

\subsection{Existence and uniqueness of continuous solutions}

Naturally, we wish to assure ourselves that the model we have constructed does indeed have a solution, preferably a unique solution. In order to establish this we adapt a result from the text by Driver [11].

Theorem 1. The delay differential equation system defined by the equations (10), (11), under the conditions that $\min \left\{\tau_{1}, \tau_{2}\right\}>0$, and $\varphi(t)$ is continuous on $[0, \tau]$ possesses a unique, continuous solution $V(t)$ on some finite interval $[0, T)$, where $T \in[0, \infty)$.

Proof of Theorem 1: For $\tau \leq t \leq 2 \tau_{1}$, we have

$$
V^{\prime}(t)=\alpha \varphi^{\frac{2}{3}}\left(t-\tau_{1}\right)-\beta \varphi\left(t-\tau_{2}\right),
$$

coupled with the initial condition $V(\tau)=\varphi(\tau)$. The right hand side of equation (10) is continuous on $D \subseteq \mathbb{R}$ and so (12) coupled with the initial condition, uniquely determines

$$
V(t)=\varphi(\tau)+\int_{\tau}^{t}\left(\alpha \varphi^{\frac{2}{3}}\left(s-\tau_{1}\right)-\beta \varphi\left(s-\tau_{2}\right)\right) d s
$$

for $\tau \leq t \leq 2 \tau$, provided $2 \tau<T$ and $V(t)$ remains in $D$. Since we now know $V(t)$ for $\tau \leq t \leq 2 \tau$ we can obtain $V(t)$ for $2 \tau \leq t \leq 3 \tau$, providing $3 \tau<T$ and $V(t)$ remains in $D$, as before. This is the traditional method of steps approach and it continues until we reach $T$ or until $V(t)$ leaves $D$. This concludes the proof $\square$.

\subsection{Boundedness of solutions}

Now that we have established the existence of a unique, continuous solution we can consider the qualitative behaviour of such a solution. This is more problematic for our system (10), (11); the presence of the nonlinear $V^{\frac{2}{3}}$ term means we cannot utilise the traditional techniques used, for example, in [5] to analyse solutions and points of equilibria for Gomptertz-type models and models based upon the logistic equation. We note that Burton [7], whilst studying a similar delay differential equation, describes such a problem as formidable. It is this work of Burton [7] (on pages 266-272), which originally appeared as part of some joint work with Furumochu [8] that we turn to for inspiration in tackling the question of qualitative behaviour of solutions. We adapt the theory for their similar problem, in order to derive a result relating to the boundedness of solutions for the model under consideration here.

To begin, we state the fixed point theorem that is the cornerstone of the result, Krasnoselskii's fixed point theorem. 
Theorem 2. ([13]) Let $\mathcal{M}$ be a closed convex non-empty subset of a Banach space $(\mathcal{S}, \| \cdot$ $\|)$. Suppose that $A$ and $B$ map $\mathcal{M}$ into $\mathcal{S}$ such that

(i) $A x+B y \in \mathcal{M} \forall x, y \in \mathcal{M}$,

(ii) $A$ is continuous and $A \mathcal{M}$ is contained in a compact set,

(iii) $B$ is a contraction with constant $a<1$.

Then there is a $y \in \mathcal{M}$ with $A y+B y=y$.

Now we state the theorem which asserts, under particular conditions, that our model possesses bounded solutions.

Theorem 3. Suppose that there exist positive constants $b, K$ such that:

$$
\begin{gathered}
b \leq K, \\
0<b<1, \\
\frac{\alpha}{\beta} \leq b, \\
2 \beta \tau_{2}+b<1 .
\end{gathered}
$$

If a given continuous initial function $\varphi$ is sufficiently small then the unique, continuous solution $V(t)$ of the system (10), (11) on $[0, \infty)$ satisfies $|V(t)|<1$.

Proof of Theorem 3: We start with some preliminaries. First assume that the initial function $\varphi:[0, \tau] \rightarrow[0, \infty)$ satisfies the restriction that $\varphi(t) \leq \Phi$, where $0<\Phi<1$.

Now let $h:[0, \infty) \rightarrow[1, \infty)$ be a strictly increasing and continuous function such that $h(0)=1, h(s) \rightarrow \infty$ as $s \rightarrow \infty$, with constant $a<1$ such that

$$
\frac{2 \beta}{h(t)} \int_{t-\tau_{2}}^{t} h(s) d s \leq a .
$$

Combining the conditions (17) and (18) yields the condition

$$
2 \beta \tau_{2}<1 .
$$

Let $\left(\mathcal{S},|\cdot|_{h}\right)$ be the Banach space of continuous functions $\psi:[0, \infty) \rightarrow \mathbb{R}$ with $|\psi|_{h}:=\sup _{t \geq 0}\left|\frac{\psi(t)}{h(t)}\right|<\infty$. Let $\left(\mathcal{M},|\cdot|_{h}\right)$ be the complete metric space of $\psi \in \mathcal{S}$ such that $|\psi(t)| \leq 1$ for $0 \leq t<\infty$ and $\psi(t)=\varphi(t)$ on $[0, \tau]$.

We rewrite (10) as

$$
V^{\prime}(t)=\alpha V^{\frac{2}{3}}\left(t-\tau_{1}\right)-\beta V(t)+\beta \frac{d}{d t} \int_{t-\tau_{2}}^{t} V(s) d s
$$

and apply the Variation of Parameters formula to obtain

$$
V(t)=V(\tau) e^{-\beta(t-\tau)}+\int_{\tau}^{t} e^{-\beta(t-s)}\left(\beta \frac{d}{d s} \int_{s-\tau_{2}}^{s} V(u) d u+\alpha V^{\frac{2}{3}}\left(s-\tau_{1}\right)\right) d s .
$$


Applying integration by parts to this expression yields

$$
\begin{aligned}
V(t)= & V(\tau) e^{-\beta(t-\tau)}+\beta \int_{t-\tau_{2}}^{t} V(u) d u-\beta e^{-\beta(t-\tau)} \int_{\tau-\tau_{2}}^{\tau} V(u) d u \\
& -\beta^{2} \int_{\tau}^{t} e^{-\beta(t-s)} \int_{s-\tau_{2}}^{s} V(u) d u d s+\alpha \int_{\tau}^{t} e^{-(t-s)} V^{\frac{2}{3}}\left(s-\tau_{1}\right) d s .
\end{aligned}
$$

Now we define the required mappings for addressing the conditions of Krasnoselskii's theorem. Define $A, B: \mathcal{M} \rightarrow \mathcal{M}$ such that, for $\psi \in \mathcal{M}$,

$$
(A \psi)(t)=\alpha \int_{\tau}^{t} e^{-\beta(t-s)} \psi^{\frac{2}{3}}\left(s-\tau_{1}\right) d s
$$

and

$$
\begin{aligned}
(B \psi)(t)= & V(\tau) e^{-\beta(t-\tau)}+\beta \int_{t-\tau_{2}}^{t} \psi(u) d u-\beta e^{-\beta(t-\tau)} \int_{\tau-\tau_{2}}^{\tau} \varphi(u) d u \\
& -\beta^{2} \int_{\tau}^{t} e^{-\beta(t-s)} \int_{s-\tau_{2}}^{s} \psi(u) d u d s .
\end{aligned}
$$

We tackle the first condition of Krasnoselskii's theorem and show that, for $\psi, \eta \in \mathcal{M}$ we have $A \psi+B \eta \in \mathcal{M}$. Let $\|\cdot\|$ be the supremum norm on $[0, \infty)$ of $\psi \in \mathcal{S}$ if $\psi$ is bounded. Then we have

$$
\begin{aligned}
|(A \psi)(t)+(B \eta)(t)| \leq & \mid \alpha\|\psi\|^{\frac{2}{3}} \int_{\tau}^{t} e^{-\beta(t-s)} d s+V(\tau) e^{-\beta(t-\tau)}+\beta\|\eta\| \int_{\tau-\tau_{2}}^{t} d u \\
& +\beta e^{-\beta(t-\tau)}\|\varphi\| \int_{\tau-\tau_{2}}^{\tau} d u+\beta^{2}\|\eta\| \int_{\tau}^{t} e^{-\beta(t-s)} \int_{s-\tau_{2}}^{s} d u d s \mid \\
\leq & \left|b\|\psi\|^{\frac{2}{3}}+\|\varphi\| e^{-\beta(t-\tau)}\left(1+\beta e^{-\beta(t-\tau)}\right)+2 \beta \tau_{2}\|\eta\|\right| \\
\leq & \left|\|\varphi\| e^{-\beta(t-\tau)}\left(1+\beta e^{-\beta(t-\tau)}\right)+2 \beta \tau_{2}+b\right| \\
< & 1
\end{aligned}
$$

for sufficiently small $\|\varphi\|$.

Moving on to the second condition of Krasnoselskii's Theorem, we show that $A$ is a continuous mapping. This is done by showing that, for $\epsilon>0$ given and $\psi, \eta \in \mathcal{M}$, we can find $\delta>0$ such that $|\psi-\eta|_{h}<\delta$ implies that $|A \psi-A \eta|_{h}<b \epsilon$. First, we take a fixed $T>0$ such that $\frac{4}{h(T)}<\epsilon . V^{\frac{2}{3}}$ is uniformly continuous on $[-1,1]$ so for $\epsilon<0$ there exists $\delta>0$ such that $\frac{\left|V_{1}-V_{2}\right|}{h(T)}<\delta$ implies that $\left|V_{1}^{\frac{2}{3}}-V_{2}^{\frac{2}{3}}\right|<\frac{\epsilon}{2}$. Thus for $|\psi(t)-\eta(t)|<\delta h(t)$, 
and for $t<T$ we have

$$
\begin{aligned}
\frac{|(A \psi)(t)-(A \eta)(t)|}{h(t)} \leq & \frac{1}{h(t)} \alpha \int_{\tau}^{t} e^{-\beta(t-s)}\left|\psi^{\frac{2}{3}}\left(s-\tau_{1}\right)-\eta^{\frac{2}{3}}\left(s-\tau_{1}\right)\right| d s \\
\leq & \frac{1}{h(t)}\left(\alpha \int_{\tau}^{T} e^{-\beta(t-s)}\left|\psi^{\frac{2}{3}}\left(s-\tau_{1}\right)-\eta^{\frac{2}{3}}\left(s-\tau_{1}\right)\right| d s+\right. \\
& \left.2 \alpha \int_{T}^{t} e^{-\beta(t-s)} d s\right) \\
\leq & \frac{1}{h(t)}\left(\frac{\alpha \epsilon}{2 \beta}\left(e^{-\beta(t-T)}-e^{-\beta(t-\tau)}\right)+\frac{2 \alpha}{\beta}\left(1-e^{-\beta(t-T)}\right)\right) \\
\leq & \frac{b \epsilon}{2}+\frac{2 b}{h(T)} \\
& <b \epsilon .
\end{aligned}
$$

Thus, we have $A$ is continuous. Now we show the equicontinuity property. If $\psi \in \mathcal{M}$ and if $\tau \leq t_{1}<t_{2}$ with $t_{2}-t_{1}<\min \left[1, \tau_{2}\right]$ then

$$
\begin{aligned}
\left|(A \psi)\left(t_{2}\right)-(A \psi)\left(t_{1}\right)\right|= & \mid \alpha \int_{t_{1}}^{t_{2}} e^{-\beta\left(t_{2}-s\right)} \psi^{\frac{2}{3}}\left(s-\tau_{1}\right) d s \\
& +\alpha \int_{\tau}^{t_{1}}\left(e^{-\beta\left(t_{2}-s\right)}-e^{-\beta\left(t_{1}-s\right)}\right) \psi^{\frac{2}{3}}\left(s-\tau_{1}\right) d s \mid \\
\leq & b \beta\left(t_{2}-t_{1}\right)+\alpha \int_{\tau}^{t_{1}}\left|e^{-\beta\left(t_{2}-\tau\right)}-e^{-\beta\left(t_{1}-\tau\right)}\right| e^{\beta(s-\tau)} d s \\
\leq & 2 b \beta\left(t_{2}-t_{1}\right) \\
\leq & 2 b K\left|t_{2}-t_{1}\right|
\end{aligned}
$$

as required. To complete our demonstration that the second condition is satisfied we show that $A \mathcal{M}$ resides in a compact set, using a technique appearing in Burton's text [6] (pages 171-172). Let $\left\{\psi_{n}\right\}$ be a sequence in $\mathcal{M}$. We can see from the definition of $|\cdot|_{h}$ that if $\left\{\psi_{n}\right\}$ converges uniformly to some function $\psi$ on compact subsets of $[0, \infty)$ then $\left|\psi_{n}-\psi\right|_{h} \rightarrow 0$ as $n \rightarrow \infty$. So now consider $\psi$ on $[0,1]$. It is uniformly bounded and, as we have seen, equicontinuous so there exists a subsequence $\left\{\psi_{n}^{1}\right\}$ converging uniformly to some continuous function $\psi$ on $[0,1]$. If we now consider $\left\{\psi_{n}^{1}\right\}$ on $[0,2]$ we again have a subsequence $\left\{\psi_{n}^{2}\right\}$ converging uniformly to a continuous function $\psi$ on [0,2]. Iterating in this manner will generate a subsequence, say $\left\{\psi_{n}^{n}\right\}$ of $\left\{\psi_{n}\right\}$, which converges uniformly on compact subsets of $[0, \infty)$ to a function $\psi \in \mathcal{M}$. Thus $M$ is compact.

Moving on to the third and final condition of Krasnoselskii's thoeorem, we show that 
$B$ is a contraction:

$$
\begin{aligned}
\left|\left(B \psi_{1}\right)(t)-\left(B \psi_{2}\right)(t)\right|_{h}= & \frac{1}{h(t)} \mid \beta \int_{t-\tau_{2}}^{t}\left(\psi_{1}(u)-\psi_{2}(u)\right) d u \\
& +\beta^{2} \int_{\tau}^{t} e^{-\beta(t-s)} \int_{s-\tau_{2}}^{s}\left(\psi_{1}(u)-\psi_{2}(u)\right) d u d s \mid \\
\leq & \beta \tau_{2}\left|\psi_{1}-\psi_{2}\right|_{h}+\beta \tau\left|\psi_{1}-\psi_{2}\right|_{h} e^{-\beta(t-s)} \\
\leq & 2 \beta \tau\left|\psi_{1}-\psi_{2}\right|_{h} \\
\leq & a\left|\psi_{1}-\psi_{2}\right|_{h}
\end{aligned}
$$

for some $a<1$ as required.

Thus, the conditions of Krasnoselskii's fixed point theorem are satisfied and our proof is concluded. $\square$

\subsection{Positivity of solutions}

Solutions $V(t)$ to the system formed by equations (10), (11) on $[0, \infty)$ must be positive in order to be meaningful from a biological viewpoint; it is impossible to have a negative number of cells and a zero solution is meaningless from the point of view that it indicates the absence of any tumour whose growth we are attempting to model.

By rewriting equation (21) in the equivalent form below

$V(t)=V(\tau) e^{-\beta(t-\tau)}+\beta \int_{\tau}^{t} e^{-\beta(t-s)}\left(V(s)-V\left(s-\tau_{2}\right)\right) d s+\alpha \int_{\tau}^{t} e^{-\beta(t-s)} V^{\frac{2}{3}}\left(s-\tau_{1}\right) d s$

we see that (due to the first and third terms on the right hand side being positive) the following inequality must be satisfied in order to guarantee a positive solution $V(t)$ :

$$
V(\tau) e^{-\beta(t-\tau)}+\alpha \int_{\tau}^{t} e^{-\beta(t-s)} V^{\frac{2}{3}}\left(s-\tau_{1}\right) d s \geq \beta \int_{t}^{\tau} e^{-\beta(t-s)}\left(V(s)-V\left(s-\tau_{2}\right)\right) d s .
$$

It is only the second term in (25) that may not be positive and we can see from its structure that $\tau_{2}$ is a crucial parameter. Letting $\tau_{2} \rightarrow 0$ we see that the right hand side of the inequality likewise tends to zero. As such, whilst we cannot obtain an explicit expression for the maximum value of $\tau_{2}$ to preserve positivity, we see that, in practical terms when trying to fit the model to data, we should not allow $\tau_{2}$ to grow too large.

\section{Fitting the model to data}

The article by Bodnar and Foryś [5] includes 6 small data sets, originally published in [16]. Each data set specifies the total number of EAT cells measured during the experiment and so the solution to our model is considered to be a function of the total cells. Time is measured in days and represents the age of the transplanted tumour. We will use this same data in order to compare our model to those discussed in [5] and to gain insight into the suitability of our system for modelling spherical tumour growth. For convenience the data is re-presented in table 1. The data in the table, when graphed makes it clear that there is some oscillatory behaviour occurring; the traditional ordinary 


\begin{tabular}{r|rrrrrr}
\hline & \multicolumn{7}{|c}{ Data Set } \\
Time(days) & 0 & 1 & 2 & 3 & 4 & 5 \\
\hline 0 & 4 & & & & & \\
2 & 8.46 & 0.6 & 0.4 & 0.3 & 0.2 & 0.1 \\
3 & 19.9 & & & & & \\
4 & 31.4 & 1.4 & 2.5 & 1.4 & 2.5 & 2.3 \\
5 & 53.6 & & & & & \\
6 & 69.2 & & & & & \\
7 & 91.2 & 8.1 & 8.3 & 7.6 & 8.2 & 8.3 \\
9 & 132 & 43.2 & 29.9 & 51.0 & 53.3 & 53.7 \\
11 & 142 & 92.9 & 139.5 & 96.3 & 102.3 & 104.3 \\
13 & 130 & & & & & \\
14 & 132 & 104.1 & 131.4 & 99.0 & 99.0 & 91.9 \\
15 & 114 & & & & & \\
16 & & 103.5 & 95.5 & 98.0 & 89.5 & 93.5 \\
17 & 81.5 & & & & & \\
18 & & 94.6 & 69.7 & 91.2 & 80.9 & 77.7 \\
21 & & 73.0 & 81.1 & 71.4 & 111.3 & 71.8 \\
23 & & 71.8 & 67.6 & 104.0 & 89.7 & 62.5 \\
25 & & 77.8 & 84.7 & 134.2 & 56.2 & \\
\hline
\end{tabular}

Table 1: Previously published tumour data [16], giving total number of EAT cells counted $\times 10^{7}$ per stated day, for each data set

differential equation models discussed in the first section are incapable of replicating such behaviour and so the idea of pursuing delay models is vindicated.

To facilitate comparisons we take a similar approach to the methodology used in [5] for generating solutions to the model. We take our initial function $\varphi(t)$ on $[0, \tau]$ to be the exact solution to equation (10) without delay; i.e. the analytic solution of equation (4). For convenience we state this initial function now:

$$
V(t)=\left(\frac{\alpha}{\beta}+\left(V_{0}^{\frac{2}{3}}-\frac{\alpha}{\beta}\right) e^{-\frac{\beta}{3} t}\right)^{3}, t \in[0, \tau],
$$

where $V_{0}=V(0)$ is the initial condition to (4) and is a parameter to also be estimated.

Bodnar and Foryś [5] use a least squares approach to fit the data to the model, estimating a maximum of four parameters (the initial condition for the ordinary differential equation which generates the initial function, two constant parameters within the delay differential equation and the single fixed delay value). It is unclear which algorithm is used in their paper to estimate the parameters in order to obtain a low measure of error; we use the term low rather than minimum because Bodnar and Foryś [5] say that "algorithms of finding minimum are unclear". They say that, for their choice of algorithm, there is no guarantee that the found minimum is global rather than local. They try to minimize this by rerunning the algorithm from different starting points but acknowledge that better fittings may be found for their models. It is also important to note that they are particularly interested in reproducing the shape of the data and so focusing on ensuring the fit minimizes the error absolutely appears not to be the priority, or appropriate. 
We do not know the standard deviation of the experimental error and so we can either assume a constant standard deviation for each data point:

$$
E=\frac{1}{N} \sqrt{\sum_{i=1}^{N}\left(V_{i}-V\left(t_{i}\right)\right)^{2}},
$$

where $N$ is the number of data points, the $V_{i}$ are the experimental values and $V\left(t_{i}\right)$ are the values predicted by the model, or assume that it is proportional to the measured value:

$$
E=\frac{1}{N} \sqrt{\sum_{i=1}^{N} \frac{\left(V_{i}-V\left(t_{i}\right)\right)^{2}}{V_{i}^{2}}} .
$$

The above alternative error formulae can therefore be thought of as a choice between measuring the error in absolute terms (28) or measuring the relative error (29). Bodnar and Foryś [5] assert that the second approach places too much emphasis on the earlier data points, which are relatively small for most of the data sets.

When fitting the data to the model (10), (11) we note a number of problems that must be overcome. Firstly, we note that the article by Baker and Paul [1] alerts its readers to hazards in using 'black box' type routines which aim to minimize some objective function in order to obtain a minimum point. Solutions to delay differential equations can contain discontinuities in their derivatives and therefore the objective function being used to minimize the error function may not be smooth. We therefore avoid such an approach.

Our first approach was a brute force approach of starting with a very large 5dimensional parameter space $\left(\alpha, \beta, \tau_{1}, \tau_{2}, V_{0}\right)$ divided into a very coarse mesh. We would then compute numerical solutions to the model (10), (11) at each of the points on the mesh, estimating the error for each mesh point. This is an estimate since for $t \in(\tau, 25]$ we are approximating the solution to the model with a numerical method - Euler's method, which is a low-order easy-to-implement scheme. Using our results to guide us, we would choose a subset of the parameter space in which to investigate further, subdivided into a finer mesh than previously used. We have applied this process for each error measure (28) and (29). In addition, for each of these, we have conducted the experiment twice: the first time without any recourse to parameter conditions and secondly by applying the conditions identified in Theorem 3, as well as checking for positivity of solutions over the time intervals within the data set. When testing conditions and positivity we rejected points in the parameter space which yielded negative values for $V$ or broke the sufficiency conditions.

Given that we are dealing with small data sets and that we do not know the standard deviation of the experimental error, we must acknowledge that the confidence intervals for our estimated parameter values are larger than perhaps one would like.

We discover that using the absolute error (28) coupled with a freedom to ignore the parameter conditions, for Data Set 0 yields a very good fit both in terms of the shape of the data and the error size (see figure 1), with the corresponding parameter values stated in the first row of table 2; here we also identify which model performed best in [5], along with its published absolute error. Furthermore, if we extend the range of $t$ we see that positivity is maintained; whilst we cannot test this for $t \in(0, \infty)$ we think that 
going to beyond ten times the length of the life of the tumour and host is sufficient. This is demonstrated in figure 2. To test the sufficient conditions of Theorem 3 in practice, means effectively testing that $\alpha<\beta$ and $2 \beta \tau_{2}<1$. We see that whilst the first of these is true, we break the second condition since $2 \beta \tau_{2}=3.159$. Figure 2 however suggests that we do have bounded solutions and that the sufficiency conditions are therefore not that strong.

Similar experiments with the remaining data sets (again, using absolute error and ignoring the theoretical conditions) are less successful, with the solutions seemingly out of phase. This seems to be due to the solution growing too quickly over the first few days. If we inspect the data sets we see that set 0 is rather different to the others, with sets 1 through 5 having missing data for the first two days. We repeated our experiments but chose to avoid attempting to model days 0 and 1 for data sets 1 through 5 . This went some way to fixing the problem, although it still leaves unanswered how one might simulate the first two days when implenting our model for days 2 and beyond. Proceeding in this way we obtain reasonably good fits for the remaining data sets, both in terms of the absolute error value and the shape of the curve. Figures 3, 4, 5, 6 and 7 demonstrate this for data sets 1 through 5 , respectively. Table 2 contains the parameter values, absolute error and the best performing model with absolute error in [5] for each of these.

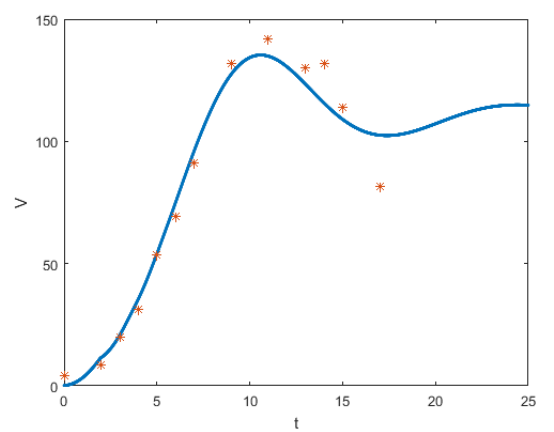

Figure 1: Data Set 0 using absolute error

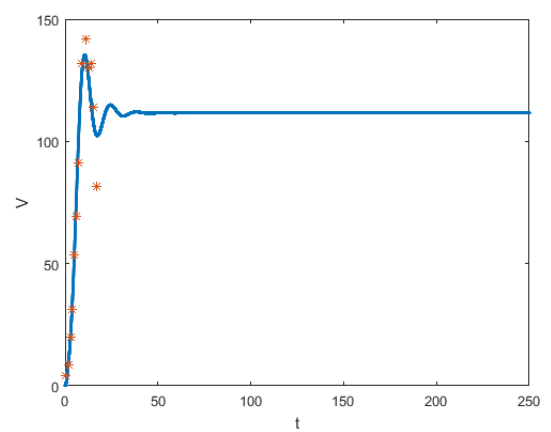

Figure 2: As figure 1 but longer time

\begin{tabular}{r|r|r|r|r|r|r|r|l} 
Data & $\alpha$ & $\beta$ & $\tau_{1}$ & $\tau_{2}$ & $V(0)$ & Error & Error [5] & Model [5] \\
\hline 0 & 0.39 & 0.81 & 1.35 & 1.95 & 0.1 & 2.085 & 6.0987 & Gompertz (one delay) \\
1 & 0.46 & 1.05 & 1.55 & 1.75 & 0.00001 & 4.857 & 7.7521 & Greenspan (one delay) \\
2 & 0.42 & 0.90 & 1.35 & 1.95 & 0.00001 & 8.3002 & 10.1939 & Gompertz (one delay) \\
3 & 0.46 & 1.03 & 1.55 & 1.85 & 0.00001 & 6.2511 & 13.0495 & Greenspan (two delays) \\
4 & 0.47 & 1.05 & 1.55 & 1.75 & 0.00001 & 5.2757 & 10.3337 & Greenspan (two delays) \\
5 & 0.47 & 1.05 & 1.55 & 1.95 & 0.00001 & 6.844 & 6.53581 & Gompertz (one delay)
\end{tabular}

Table 2: Parameter estimates and associated absolute errors, minimum error quoted in [5] and associated model. Errors are calculated using (28).

We repeated our experiments for absolute error by insisting that the conditions of Theorem 3 are met. This had the result of being too restrictive on the set of parameter 


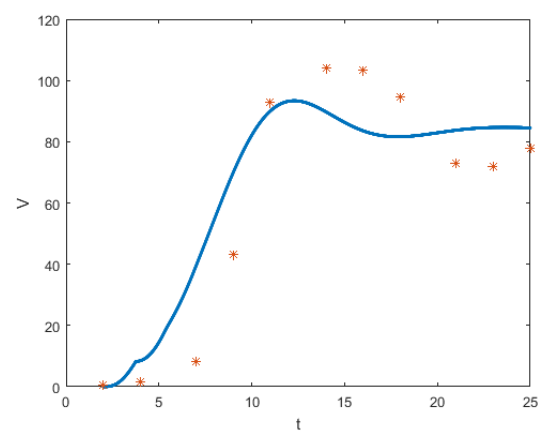

Figure 3: Data Set 1 (day 2 onwards) using absolute error

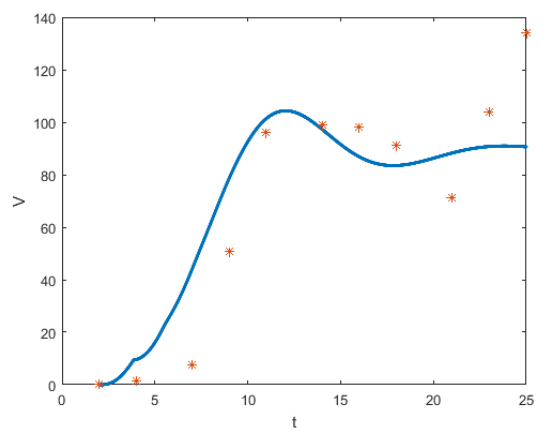

Figure 5: Data Set 3 (day 2 onwards) using absolute error

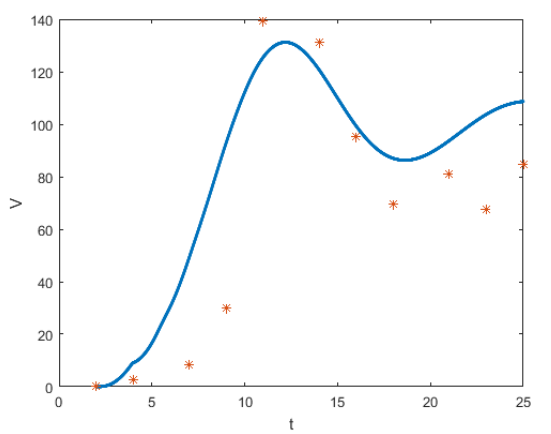

Figure 4: Data Set 2 (day 2 onwards) using absolute error

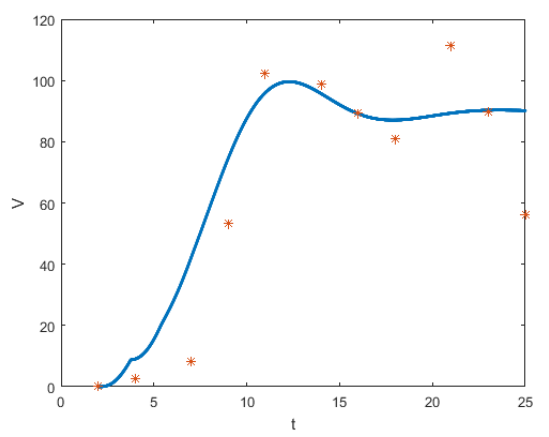

Figure 6: Data Set 4 (day 2 onwards) using absolute error

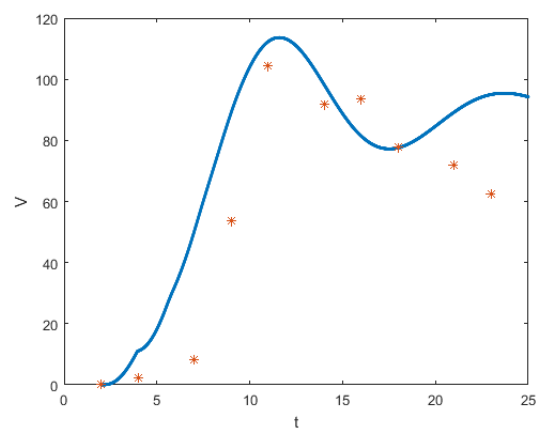

Figure 7: Data Set 5 (day 2 onwards) using absolute error

values and the oscillatory behaviour was lost. We also attempted experiments with the relative error but we had the problem identified by Bodnar and Forys̀ [5] that too much emphasis is placed on the early data points (where we have already identified a problem), and so we again lose oscillatory behaviour. Figures 8 and 9 demonstrate both of these problems respectively, where both problems manifest themselves in a way which gives a 
good fit for the first half of the data but fails once the data starts to exhibit oscillations.

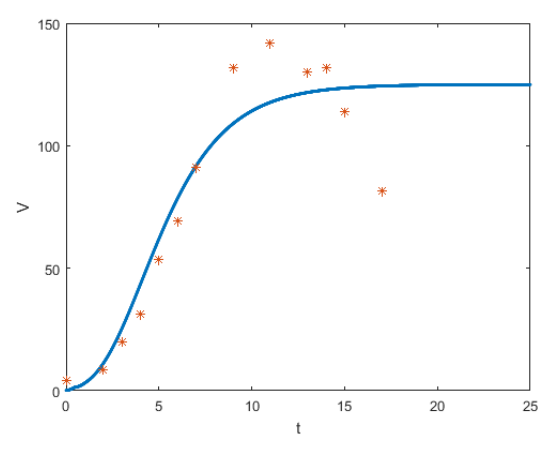

Figure 8: Data Set 0, Theorem 3 satisfied

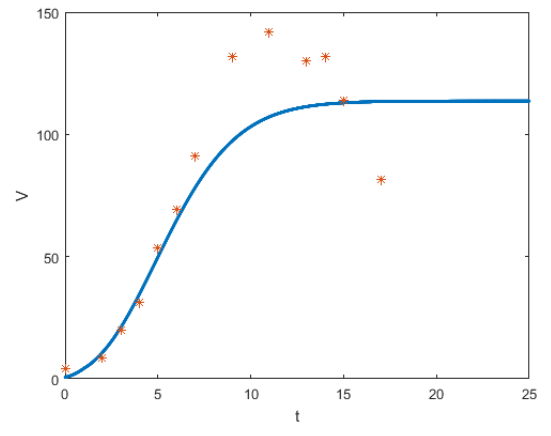

Figure 9: Data Set 0, relative error (29) used to estimate parameters

To complete our numerical experiments we applied a Monte-Carlo type approach to estimating parameters for data set 0 . Our approach was as follows. We would start with our initial, large parameter space but use a finer mesh. Obviously, due to the impractical computation time we could not compute approximate solutions at every point on the mesh so instead we randomly chose a number of points (using a uniform distribution since we have no additional insight into the problem to believe that one subset of the space would be a better choice than any other). We would then rank these solutions by error (28), looking at the minimum and maximum parameter values appearing in a top subset of this ranking list. This would then take us to a subset of the parameter space, on which we would repeat the process in an iterative manner in order to seek a refinement.

We then obtained a set of parameter values which yield the solution in figure 10 . This solution is an exceptionally good fit for the data, both in terms of producing a small absolute error and reproducing the oscillatory behaviour. Figure 11 also shows the solution remains positive and bounded for $t$ values well beyond the final day for which we have data. This also highlights how large the computational task is in terms of obtaining a solution that both produces a small error and reproduces the shape of the data. If we look at the parameter values identified by the Monte-Carlo approach, they are some distance away from those identified previously within the 5-dimensional parameter space. It serves to remind us of the danger of closing in on local minima rather than global minima within the space and the computational time required to do this. Furthermore, it highlights that the gap between the theoretical work and the experimental work.

\section{Concluding Remarks}

We have demonstrated, through numerical experiments that our choice of Bertalanffy's model with two distinct delays can perform as well (and sometimes better) than other models under consideration by researchers. We have positively addressed the assertion by Gerlee [12] that the Bertalanffy model is worthy of further consideration; Vaidya 


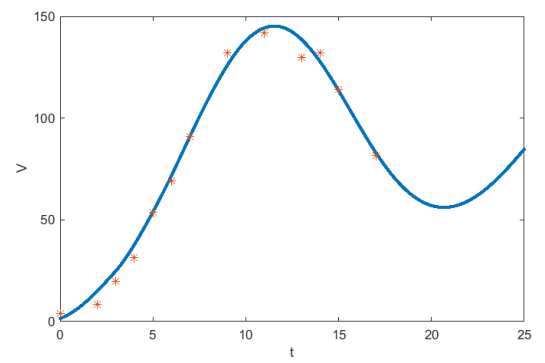

Figure 10: Data Set 0, Monte-Carlo approach $\alpha=3.2961, \beta=0.7174, \tau_{1}=1.995, \tau_{2}=2.94$, $V(0)=1.5714$, Error $=1.2452$

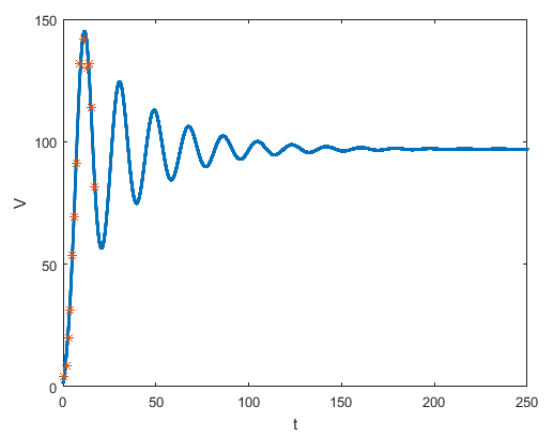

Figure 11: Data Set 0, Monte-Carlo based approach computed for longer time

and Alexandro Jr. [18] found instances, in the non-delay case, where the Bertlanffy model outperforms other models and we have now shown that this situation continues when delays are introduced. We have addressed some of the theoretical issues around model choice, such as existence, uniqueness and behaviour of solutions to the model, making use of the work by Burton and Furumochu [8] and adapting it to our circumstances. For the small data sets we have used, we have seen that the absolute error has been a better tool for attempting to obtain solutions which reproduce the shape of the data. Interestingly, we note that table 2 shows our parameters are in close proximity to one another from data set to data set; for example all of the $\alpha$ values are in the interval $[0.39,0.47]$ yet the Monte Carlo approach produces an $\alpha$ value further away. Therefore we must be careful when jumping to conclusions about the error within our parameter estimates.

Our chosen model is more complex then those discussed in [5]; it has five parameters rather than four (by virtue of having two distinct delays) and it is a nonlinear system that does not satisfy a Lipschitz condition, thereby limiting the number of theoretical tools available to us for studying the qualitative behaviour of the solutions. The pay-off for introducing such complexities is the quality of the results.

\section{Acknowledgements}

The authors wish to thank the University of Princess Nourah bint Abdulrahman, Saudi Arabia for supporting the visit of Dr Asmaa Al Themairi to the University of Chester, which facilitated this collaboration. The authors would also like to thank the organisors of the Fifth International Workshop on Analysis and Numerical Approximation of Singular Problems, Lagos, Portugal 2015, for giving Dr Jason Roberts the opportunity to present this work. We would also like to thank those delegates who attended the workshop and demonstrated their interest in this work by attending the presentation and providing insight comments and suggestions. Finally, we thank the reviewers for their helpful comments. 


\section{References}

[1] C.T.H. Baker, C.A.H. Paul, Pitfalls in parameter estimation for delay differential equations, SIAM J. Sci. Comput. 18(1) (1997) 305-314.

[2] R.E. Bellman, K.L. Cooke, Differential-Difference Equations, Academic Press, New York 1963.

[3] L. Bertalanffy, Problems of organic growth, Nature, 163(4135) (1949) 156-158.

[4] P. Bi, S. Ruan, Bifurcations in delay differential equations and applications to tumor and immune system interaction models, SIAM J. Applied Dynamical Systems 12(4) (2013) 1847-1888.

[5] M. Bodnar, U. Foryś, Three types of simple DDEs describing tumour growth, J. Biol. Syst. 15(4) (2007), 453-471.

[6] T.A. Burton, Stability and Periodic Solutions Of Ordinary And Functional Differential Equations, Dover, New York, 2005.

[7] T.A. Burton, Stability by Fixed Point Theory for Functional Differential Equations, Dover, New York, 2006.

[8] T.A. Burton, T. Furumochu, Asymptotic behavior of solutions of functional differential equations by fixed point theorems, Dynam. Systems Appl. 11 (2002) 499-519.

[9] F. Brauer, C. Castillo-Chavez, Mathematical Models in Population Biology and Epidemiology, second ed., Springer, New York 2011.

[10] H.M. Byrne, The Effect of Time Delays on the Dynamics of Avascular Tumor Growth, Mathematical Biosciences 144 (1997), 83-117.

[11] R.D. Driver, Introduction to Ordinary Differential Equations, Harper \& Row, New York, 1978.

[12] P. Gerlee, The model muddle: in search of tumour growth laws, Journal of Cancer Research 73(8) (2013), 2407-11.

[13] M.A. Krasnoselskii, Some problems of nonlinear analysis, Amer. Math. Soc. Transl. 10(2) (1958), 345-409.

[14] M. Marušić, Z̆.Bajzer, J.P. Freyer, S. Vuk-Pavlović, Analysis of growth of multicellular tumour spheroids by mathematical models, Cell Prolif. 27(2) (1994), 73-94.

[15] J.D. Murray, Mathematical Biology, second ed., Springer-Verlag, Berlin, 1993.

[16] R. Schuster, H. Schuster, Reconstruction models for the Ehrlich Ascites tumor for the mouse, in: O. Arino, D. Axelrod, M. Kimmel (Eds.), Mathematical Population Dynamics Volume 2, Wuertz, 1995, pp. 335-348.

[17] H. Smith, An Introduction to Delay Differential Equations with Applications to the Life Sciences, Springer, New York, 2011.

[18] G. Vaidya, F.J. Alexandro Jr, Evaluation of some mathematical models of tumor growth, Int. J. Bio-Medical Computing, 13(1) (1982), 19-35. 